\title{
RESEARCH ARTICLE \\ Optimal consumption and portfolio choice with ambiguous interest rates and volatility
}

\author{
Qian $\operatorname{Lin}^{1} \cdot$ Frank Riedel ${ }^{2,3}$ (DD
}

Received: 5 February 2020 / Accepted: 24 August 2020 / Published online: 10 September 2020

(c) The Author(s) 2020

\begin{abstract}
We study continuous-time consumption and portfolio choice in the presence of Knightian uncertainty about interest rates. We develop the stochastic model that involves singular priors and analyze optimal behavior. When there is sufficiently large uncertainty about interest rates, the agent invests in the asset market only and abstains from the bond market.
\end{abstract}

Keywords Portfolio Choice $\cdot$ Knightian Uncertainty $\cdot$ Model uncertainty · Interest Rate Ambiguity

JEL Classification D81 - G11 - G12

\section{Introduction}

Optimal consumption and portfolio decisions play a fundamental role for individual investors, pension funds, and insurance companies alike. Life-cycle models also form the basic building block for more complex economic models that are used in economic policy and governance discussions. In this paper, we consider an otherwise standard

This work is supported by the National Natural Science Foundation of China (No. 11971364), and the German Research Foundation (DFG) via Grant Ri-1128-7-1 and the CRC 1283.

Frank Riedel

frank.riedel@uni-bielefeld.de

Qian Lin

linqian@whu.edu.cn

1 School of Economics and Management, Wuhan University, Wuhan, China

2 Center for Mathematical Economics, Bielefeld University, Bielefeld, Germany

3 Department of Economic and Financial Sciences, University of Johannesburg, Johannesburg, South Africa 
life-cycle consumption and portfolio problem for an investor who faces Knightian uncertainty about interest rates. ${ }^{1}$

Interest rates vary considerably over time. Equilibrium interest rates depend on the economy's growth, the volatility of the market, and agents' preference parameters as subjective discount rate, risk aversion, and intertemporal rate of substitution. All these parameters are hard to predict or estimate in the long run. While investors were used to high levels of interest rates in the 1970s when the treasury rate in the US, e.g., fluctuated around $10 \%$, we have by now an extended period of very low interest rates around the zero level. The level of interest rates is strongly influenced by central bank policies. Last not least, inflation adds an additional flavor of uncertainty that is difficult to model or predict.

For a long term investor, there is thus no riskless asset. While the consumption and portfolio choice problem for investors who face Knightian uncertainty about the returns of risky assets has been amply studied (see below), the role of Knightian or model uncertainty of interest rates has not been tackled so far. We thus develop here a model that allows to discuss the impact of interest rate uncertainty on optimal investment and consumption decisions within the classic Samuelson continuous-time life cycle model. We take model uncertainty about the short rate and, in fact, the whole term structure, into account. We consider an investor who is willing to work with fixed bounds $\underline{r}<\bar{r}$ for the short rate. Every adapted process $\left(r_{t}\right)$ with values in the interval $[\underline{r}, \bar{r}]$ is considered as a possible trajectory at time 0 . At time $t$, the past values of the short rate $\left(r_{s}\right)_{s \leq t}$ including the current value $r_{t}$ are known, of course. The investor still faces model uncertainty about the future realizations $r_{u}$ for $u>t$. In this sense, we assume here that the ambiguity about the future short rates is persistent and no learning occurs. ${ }^{2}$

The main new finding is the following. If interest rate uncertainty is sufficiently high, it is optimal to put all wealth into risky assets. This is in sharp contrast to other studies involving uncertainty-averse investors. For example, in a pioneering study, Dow and Werlang (1992) show that ambiguity-averse investors rather shy away from risky assets when they face Knightian uncertainty about expected returns. In Dow and Werlang's one period model, the effect of interest rate uncertainty cannot be studied, of course. When there is no or only small interest rate uncertainty, a similar results holds true in our continuous-time model. However, we find it important to stress the relevance of interest rate uncertainty for long term investors.

Knightian uncertainty has recently attracted a great deal of attention, both in practice, as the sensitivity of many financial decisions with respect to questionable probabilistic assumptions became clear, and in theory, where an extensive theory of decision making and risk measurement under uncertainty has emerged. Gilboa and Schmeidler (1989) provide the foundation for a new approach to decisions under Knightian uncertainty by weakening the strong independence axiom or sure thing principle that was used previously by Savage (1954) and Anscombe and Aumann

\footnotetext{
1 We also discuss uncertainty about drift and volatility in Sect. 3 below.

2 From a conceptual perspective, the short rate is determined by independent and indistinguishable experiments in the sense of Epstein and Schneider (2003a); in every (infinitesimal) period, a new ambiguous experiment is carried out which is independent from the past to determine the next short rate. As a consequence, the agent cannot learn from past data.
} 
(1963) to justify (subjective) expected utility. The models are closely related to monetary risk measures (Artzner et al. 1999). Subsequently, the theory has been generalized to variational preferences (Maccheroni et al. 2006a; Föllmer and Schied 2002) and dynamic time-consistent models (Epstein and Schneider 2003b; Riedel 2004; Maccheroni et al. 2006b; Föllmer and Penner 2006).

Concerning portfolio and consumption choice, the pioneering results of Merton $(1969,1971)$ are still the basic reference for life-cycle consumption and portfolio choice under uncertainty. As mean return, volatility, and interest rates are known constants in Merton's model, the consequences of having stochastic, time-varying dynamics for these parameters have been studied in great detail. Mean-reverting drift (or "predictable returns"), stochastic volatility models and models with stochastic term structures have been studied in detail. These models all work under the expected utility paradigm as they assume a known distribution for the parameters; for example, Barberis (2000) studies mean-reverting returns and estimation errors. Korn and Kraft (2001) study portfolio problems with stochastic interest rates. Chacko and Viceira (2005), and Kraft (2005) allow for stochastic volatility. We refer the reader to Liu (2007) for a recent general approach with stochastic interest rates and volatilities. The typical result in this literature identifies additional terms next to the classic optimal portfolio of Merton that are related to the demand for hedging the new diffusive factors. In particular, the portfolio weights vary stochastically with the factor estimates over time.

Robust statistics and robust control as well as the decision-theoretic literature on Knightian uncertainty share a lot of formal and conceptual similarities. The typical "penalty" approach to robust control used by Hansen and Sargent (2011) can be viewed as a special case of variational preferences (Maccheroni et al. 2006a, b) where the agent uses entropy as a penalty function. Our approach of a pessimistic multiple prior model is also a special case of variational preferences but with a different penalty function. Using the robust control approach of Anderson et al. (2003), Trojani and Vanini (2002), Maenhout (2004), Luo (2017) and Luo et al. (2020) study the robust portfolio choice problem with drift ambiguity. Drift ambiguity in continuous time is also discussed in Chen and Epstein (2002), Schied (2005), Quenez (2004), Schied (2008), Miao (2009), Liu (2010, 2011) among others. Föllmer et al. (2009) survey this literature. Drift and volatility uncertainty have been studied in the recent papers Biagini and Mustafa (2017), Epstein and Ji (2013), and Neufeld and Nutz (2018). These papers all work with a known interest rate.

The paper is organized as follows. The next section formulates the interest rate ambiguity model within the new framework and states the main theorem. In Sect. 3, we formulate a general model that allows for Knightian uncertainty about drift, volatility, and interest rates, and we provide the relevant proofs.

\section{Knightian uncertainty about interest rates and the main theorem}

This paper investigates optimal consumption and investment policies under Knightian uncertainty. We extend the Samuelson model of financial markets to allow for Knightian uncertainty about interest rates in the sense that the agent does not know 
the probability distribution of future interest rates, yet is willing to work with certain bounds for the relevant parameters.

In the continuous-time diffusion framework, three parameter processes, drift (or expected return), volatility and short term interest rate, describe the dynamics of the risky asset and the bond. To make our point succinctly, we assume that volatility $\sigma>0$ and drift $\mu \in \mathbb{R}$ are known, yet the interest rate is uncertain. In general, drift and volatility uncertainty are important as well, of course. We treat the completely general case in Sect. 3 below. ${ }^{3}$

We model the Knightian uncertainty about the real interest rate by an interval $[\underline{r}, \bar{r}]$. The investor is uncertain about the distribution of the (adapted) interest rate process $r=\left(r_{t}\right)$. Yet, he is willing to take a stand on upper and lower bounds for the evolution of interest rates. We denote the set of all adapted processes with values in the interval $[\underline{r}, \bar{r}]$ by $\Theta$.

We consider a market with a risky asset and a bond. For each $r \in \Theta$, we consider asset price dynamics

$$
d \bar{P}_{t}=\bar{P}_{t} r_{t} d t, \bar{P}_{0}=1
$$

and

$$
d S_{t}=\mu S_{t} d t+\sigma S_{t} d W_{t}^{r}, S_{0}=1
$$

for bond and stock, respectively, under a probability measure $P^{r}$, where $W^{r}$ is a $P^{r}$ Brownian motion. Let $\mathcal{P}$ be the set of all probability measures $P^{r}$ constructed in this way. We call a pair $(\pi, c)$ of adapted processes a consumption-portfolio strategy if $\int_{0}^{T}\left(\pi_{s}^{2}+c_{s}^{2}\right) d s<\infty, P$-a.s., for all $P \in \mathcal{P}$. The wealth of the investor with some initial endowment $x_{0}>0$ and portfolio-consumption policy $(\pi, c)$ is given by $X_{0}=x_{0}$ and the budget dynamics

$$
d X_{t}^{(\pi, c)}=r_{t} X_{t}^{(\pi, c)}\left(1-\pi_{t}\right) d t+X_{t}^{(\pi, c)} \pi_{t} \mu d t-c_{t} d t+X_{t}^{(\pi, c)} \pi_{t} \sigma d W_{t}^{r}
$$

under $P^{r}$. The consumption-portfolio strategy $(\pi, c)$ is admissible if for all $r \in \Theta$ we have $X^{(\pi, c)} \geq 0, P^{r}$-a.s., for all $P^{r} \in \mathcal{P}$. We denote by $\Pi$ the set of admissible consumption and portfolio strategies.

Consider an ambiguity-averse agent in the spirit of Gilboa and Schmeidler (1989) who maximizes the minimal expected utility over the set of priors. The investor's utility of consuming $c$ and bequesting a terminal wealth $X_{T}^{(\pi, c)}$ is defined by

$$
U(c, X)=\inf _{P \in \mathcal{P}} E_{P}\left[\int_{0}^{T} u\left(s, c_{s}\right) d s+\Phi\left(T, X_{T}^{(\pi, c)}\right)\right],
$$

\footnotetext{
3 This paper is a completely revised and shortened version of our working paper Lin and Riedel (2014) that was the first paper on the topic where the reader can find additional material.
} 
where

$$
u(t, c)=\exp (-\delta t) \frac{c^{1-\alpha}}{1-\alpha}, \Phi(T, x)=\frac{K x^{1-\alpha}}{1-\alpha}
$$

for some $\delta>0, \alpha>0, K>0$, and $\alpha \neq 1 .^{4}$

We are now able to state our main insight concerning Knightian uncertainty about interest rates. Technical details on the general solution of the problem for other parameter constellations can be found in the next section.

Proposition 1 With sufficient ambiguity about interest rates, more precisely, if

$$
\underline{r} \leq \mu-\alpha \sigma^{2} \leq \bar{r}
$$

the investor does not participate in the money market and puts all capital into the stock.

The intuition for the above result is as follows. If the investor is convinced that investing in stocks is more profitable than keeping money in the savings account, while accounting for risk aversion, i.e., if $\underline{r} \leq \mu-\alpha \sigma^{2}$, then he does not want to stay away from the opportunities that the stock market promises. As the analysis below will show in more detail, putting all wealth into risky assets hedges the investor from interest rate uncertainty as local expected returns are then independent of the interest rate. For the identified parameters, this kind of hedging is indeed optimal for the agent.

Another way of interpreting the results is as follows. With interest rate uncertainty, it is optimal to put all wealth into the risky asset if the set of Merton rations contains 1 , i.e., there is a parameter $r$ with $\frac{\mu-r}{\alpha \sigma^{2}}=1$. In this case, due to ambiguity aversion, the investor does not want to borrow additional money in order to buy even more of the risky asset as interest rates might turn out high, nor does he want to reduce his exposure as interest rates might turn out too low.

\section{Proof of the main theorem and extensions}

In this section, we provide the proof of our main theorem. We also show that our findings are robust with respect to Knightian uncertainty about drift and volatility. We thus study now a continuous-time Samuelson model that features Knightian uncertainty about drift, volatility and interest rates. It includes the model of Sect. 2 as a special case, of course.

Let $C([0, T])^{2}$ be the set of all continuous paths with values in $\mathbb{R}^{2}$ over the finite time horizon $[0, T]$ endowed with the sup norm. Our state space is

$$
\Omega_{0}=\left\{\omega: \omega \in C([0, T])^{2}, \omega_{0}=(1,1)^{\prime}\right\}
$$

\footnotetext{
4 The solution for $\alpha=1$ which corresponds to log-utility can be easily read off our solutions by setting formally $\alpha=1$ in our formulas. The proof is easily adapted.
} 
endowed with the Borel $\sigma$-field $\mathcal{B}$. We will consider the canonical process $Y_{t}(\omega)=$ $\omega_{t}, t \geq 0$, for all $\omega \in \Omega_{0}$. Let $P_{0}$ be the classic Wiener measure, and $\mathcal{F}=\left(\mathcal{F}_{t}\right)_{t \geq 0}$ denote the filtration generated by $Y$, completed by all $P_{0}$-null sets.

In the continuous-time diffusion framework, three parameter processes, drift (or expected return), volatility and interest rate uncertainty, describe all uncertainty. We model an investor who is not sure about the distribution of the drift process $\mu=\left(\mu_{t}\right)$, about the distribution of the volatility process $\sigma=\left(\sigma_{t}\right)$, nor about the distribution of the interest rate process $r=\left(r_{t}\right)$. Knightian uncertainty is captured by a convex and compact subset $\Theta \subset \mathbb{R}^{3}$. The investor is willing to make the assumption that all $\mathcal{F}$-progressively measurable processes $\theta=\left(\mu_{t}, \sigma_{t}, r_{t}\right)_{t \geq 0}$ with values in $\Theta$ are possible trajectories for the parameter processes. In this paper, we consider the special rectangular case of $\Theta=[\mu, \bar{\mu}] \times[\underline{\sigma}, \bar{\sigma}] \times[\underline{r}, \bar{r}]$, for $\mu \leq \bar{\mu}, 0<\underline{\sigma} \leq \bar{\sigma}$ and $\underline{r} \leq \bar{r}$.

Fix $\theta=(\mu, \sigma, r) \in \Theta$. Let $P^{\theta}$ be a probability measure on $(\Omega, \mathcal{B})$ such that $Y$ solves the following stochastic differential equation

$$
d Y_{t}=\left(\begin{array}{l}
d S_{t} \\
d \bar{P}_{t}
\end{array}\right)=\left(\begin{array}{l}
\mu_{t} S_{t} d t+\sigma_{t} S_{t} d W_{t}^{\theta} \\
r_{t} \bar{P}_{t} d t
\end{array}\right),\left(\begin{array}{l}
S_{0} \\
\bar{P}_{0}
\end{array}\right)=\left(\begin{array}{l}
1 \\
1
\end{array}\right),
$$

where $W^{\theta}$ is a $P^{\theta}$-Brownian motion. ${ }^{5}$ The first coordinate of $Y$ models the asset while the second coordinate models the bond price. Let $\mathcal{P}_{0}$ be the set of all probability measures $P^{\theta}$ constructed in this way. The set of priors $\mathcal{P}$ is the closure of $\mathcal{P}_{0}$ under the topology of weak convergence.

We call a pair $(\pi, c)$ a consumption-portfolio strategy, if $(\pi, c)$ are $\mathcal{F}$-progressively measurable, and $\int_{0}^{T}\left(\pi_{s}^{2}+c_{s}^{2}\right) d s<\infty, P$-a.s., for all $P \in \mathcal{P}$. The wealth of the investor with some initial endowment $x_{0}>0$ and portfolio-consumption policy $(\pi, c)$ is given by

$$
d X_{t}^{(\pi, c)}=r_{t} X_{t}^{(\pi, c)}\left(1-\pi_{t}\right) d t+X_{t}^{(\pi, c)} \pi_{t} \mu_{t} d t-c_{t} d t+X_{t}^{(\pi, c)} \pi_{t} \sigma_{t} d W_{t}^{\theta},
$$

under $P^{\theta} \in \mathcal{P}$. The consumption-portfolio strategy $(\pi, c)$ is admissible if for all $P \in$ $\mathcal{P}, X_{t}^{(\pi, c)} \geq 0, P$-a.s., $t \in[0, T]$. We denote by $\Pi$ the set of admissible consumption and portfolio strategies.

We consider an ambiguity-averse agent who maximizes the minimal expected utility over the set of priors $\mathcal{P}$. We define the value function

$$
V\left(x_{0}\right)=\sup _{(\pi, c) \in \Pi} \inf _{P \in \mathcal{P}} E_{P}\left[\int_{0}^{T} u\left(s, c_{s}\right) d s+\Phi\left(T, X_{T}^{(\pi, c)}\right)\right] .
$$

We will solve the above problem by using the analog of the martingale optimality principle that is well known from the classical Merton problem. Let us write $O=$ $[0, T) \times \mathbb{R}_{+}$and $\bar{O}=O \cup \partial O$, where $\partial O$ is the boundary of $O$.

\footnotetext{
5 The probability measure $P^{\theta}$ can be constructed using techniques for weak solutions of stochastic differential equations and the related martingale problems, see Chapter 9 in Revuz and Yor (1999), e.g.
} 
Theorem 1 Let $\varphi \in C^{1,2}(O) \cap C(\bar{O})$ with boundary condition $\varphi(T, x)=\Phi(T, x)$ for all $x \in \mathbb{R}_{+}$. Define for any $(\pi, c) \in \Pi$

$$
M_{t}^{(\pi, c)}=\int_{0}^{t} u\left(s, c_{s}\right) d s+\varphi\left(t, X_{t}^{(\pi, c)}\right) .
$$

Suppose that the following two conditions hold true:

(i) for any $(\pi, c) \in \Pi$, there exists $Q \in \mathcal{P}$ such that $M^{(\pi, c)}$ is a $Q$-supermartingale;

(ii) there exists $\left(\pi^{*}, c^{*}\right) \in \Pi$ and $Q^{*} \in \mathcal{P}$ such that

$$
\inf _{P \in \mathcal{P}} E_{P}\left[\int_{0}^{T} u\left(s, c_{s}^{*}\right) d s+\Phi\left(T, X_{T}^{\left(\pi^{*}, c^{*}\right)}\right)\right]=E_{Q^{*}}\left[\int_{0}^{T} u\left(s, c_{S}^{*}\right) d s+\Phi\left(T, X_{T}^{\left(\pi^{*}, c^{*}\right)}\right)\right]
$$

and $M^{\left(\pi^{*}, c^{*}\right)}$ is a $Q^{*}$-martingale.

Then $\left(\pi^{*}, c^{*}\right)$ is an optimal portfolio-consumption policy, and $V\left(x_{0}\right)=\varphi\left(0, x_{0}\right)$.

Proof We will verify that for all admissible policies $(\pi, c)$, the (minimal) expected utility under Knightian uncertainty is bounded by $\varphi\left(0, x_{0}\right)$ and that the candidate optimal policy attains the upper bound.

For $(\pi, c) \in \Pi$, from $\varphi(T, x)=\Phi(T, x)$ and (i) we have

$$
E_{Q}\left[\int_{0}^{T} u\left(s, c_{s}\right) d s+\Phi\left(T, X_{T}^{(\pi, c)}\right)\right]=E_{Q}\left[M_{T}^{(\pi, c)}\right] \leq M_{0}^{(\pi, c)}=\varphi\left(0, x_{0}\right) .
$$

Therefore, we have

$$
V\left(x_{0}\right)=\sup _{(\pi, c) \in \Pi} \inf _{P \in \mathcal{P}} E_{P}\left[\int_{0}^{T} u\left(s, c_{s}\right) d s+\Phi\left(T, X_{T}^{(\pi, c)}\right)\right] \leq \varphi\left(0, x_{0}\right) .
$$

On the other side, from (ii) it follows that

$$
E_{Q^{*}}\left[\int_{0}^{T} u\left(s, c_{s}^{*}\right) d s+\Phi\left(T, X_{T}^{\left(\pi^{*}, c^{*}\right)}\right)\right]=M_{0}^{\left(\pi^{*}, c^{*}\right)}=\varphi\left(0, x_{0}\right),
$$

which yields

$$
V\left(x_{0}\right)=\sup _{(\pi, c) \in \Pi} \inf _{P \in \mathcal{P}} E_{P}\left[\int_{0}^{T} u\left(s, c_{s}\right) d s+\Phi\left(T, X_{T}^{(\pi, c)}\right)\right] \geq \varphi\left(0, x_{0}\right) .
$$

From (3.2) and (3.3) we get the desired result. The proof is complete.

In the next step, we will find the suitable martingale and the corresponding policies that solve the optimization problem. Recall that for any admissible policy $(\pi, c)$, we let $X^{(\pi, c)}$, the wealth process under $P^{\theta} \in \mathcal{P}$ is

$$
d X_{t}^{(\pi, c)}=r_{t} X_{t}^{(\pi, c)}\left(1-\pi_{t}\right) d t+X_{t}^{(\pi, c)} \pi_{t} \mu_{t} d t-c_{t} d t+X_{t}^{(\pi, c)} \pi_{t} \sigma_{t} d W_{t}^{\theta} .
$$


By Itô's lemma under $P^{\theta} \in \mathcal{P}$, we have

$$
\begin{aligned}
d M_{t}^{\pi, c}= & \left(u\left(t, c_{t}\right)-c_{t} \varphi_{x}\left(t, X_{t}^{(\pi, c)}\right)+\varphi_{t}\left(t, X_{t}^{(\pi, c)}\right)+\varphi_{x}\left(t, X_{t}^{(\pi, c)}\right)\left(r_{t} X_{t}^{(\pi, c)}\right.\right. \\
& \left.\left.+\pi_{t} X_{t}^{(\pi, c)}\left(\mu_{t}-r_{t}\right)\right)+\frac{1}{2} \pi_{t}^{2}\left(X_{t}^{(\pi, c)}\right)^{2} \sigma_{t}^{2} \varphi_{x x}\left(t, X_{t}^{(\pi, c)}\right)\right) d t \\
& +\varphi_{x}\left(t, X_{t}^{(\pi, c)}\right) \pi_{t} \sigma X_{t}^{(\pi, c)} d W_{t}^{\theta} .
\end{aligned}
$$

Since for any $M^{\pi, c}$, there exists $Q \in \mathcal{P}$ such that $M^{\pi, c}$ is a supermartingale under $Q$, and for some $M^{\pi^{*}, c^{*}}$, there exists $Q^{*} \in \mathcal{P}$ such that $M^{\pi^{*}, c^{*}}$ is a martingale under $Q^{*}$, then the supremum over $(\pi, c) \in \mathbb{R} \times \mathbb{R}_{+}$of the infimum over $(\mu, \sigma, r) \in$ $[\mu, \bar{\mu}] \times[\underline{\sigma}, \bar{\sigma}] \times[\underline{r}, \bar{r}]$ of the drift of $M^{\pi, c}$ must be zero. Therefore, these heuristics lead us to the following Hamilton-Jacobi-Bellman-Isaacs (HJBI) equation

$$
\begin{gathered}
\sup _{(\pi, c) \in \mathbb{R} \times \mathbb{R}_{+}}\left\{u(t, c)+\varphi_{t}(t, x)-c \varphi_{x}(t, x)+\inf _{r \in[\underline{r}, \bar{r}]}\left\{x r \varphi_{x}(t, x)(1-\pi)\right\}\right. \\
\left.+\inf _{(\mu, \sigma) \in[\underline{\mu}, \bar{\mu}] \times[\underline{\sigma}, \bar{\sigma}]}\left\{\varphi_{x}(t, x) x \pi \mu+\frac{1}{2} x^{2} \varphi_{x x}(t, x) \pi^{2} \sigma^{2}\right\}\right\}=0 .
\end{gathered}
$$

The next theorem will show that when there is sufficiently large uncertainty about interest rates, the agent invests in the asset market only and abstains from the bond market.

Theorem 2 If $\underline{r} \leq \mu-\alpha \bar{\sigma}^{2} \leq \bar{r}$, then the value function of the utility maximization problem has the form

$$
\varphi(t, x)=f(t) \frac{x^{1-\alpha}}{1-\alpha}
$$

for

$$
f(t)=\left[K^{\alpha^{-1}} e^{\beta \alpha^{-1}(T-t)}+\alpha(\beta-\delta)^{-1} e^{-\delta \alpha^{-1} t}\left(e^{(\beta-\delta) \alpha^{-1}(T-t)}-1\right)\right]^{\alpha},
$$

where $\beta=\left(\underline{\mu}-\frac{1}{2} \alpha \bar{\sigma}^{2}\right)(1-\alpha)$. The optimal portfolio choice is $\pi^{*}=1$ and the optimal consumption choice is

$c^{*}=\left[K^{\alpha^{-1}} e^{\beta \alpha^{-1}(T-t)}+\alpha(\beta-\delta)^{-1} e^{-\delta \alpha^{-1} t}\left(e^{(\beta-\delta) \alpha^{-1}(T-t)}-1\right)\right]^{-1} x \exp \left(-\delta \alpha^{-1} t\right)$.

Proof It is sufficient to check the conditions of Theorem 1. As usual, we will verify that for all admissible policies $(\pi, c)$, the (minimal) expected utility under Knightian uncertainty is bounded by $\varphi\left(0, x_{0}\right)$ and that the candidate optimal policy attains the upper bound.

We claim that the worst case parameters are $\mu^{*}=\underline{\mu}, \sigma^{*}=\bar{\sigma}$, and $r^{*}=\mu-\alpha \bar{\sigma}^{2}$. By assumption, $\underline{r} \leq r^{*} \leq \bar{r}$, and the corresponding candidate worst case measure $P^{*}=P^{\left(\mu^{*}, \bar{\sigma}, r^{*}\right)} \in \overline{\mathcal{P}}$. 
Denote by $\varphi(t, x)$ the value function of the classic Merton problem under the candidate worst case prior $P^{*}$ as given by Eq. (3.5). From Merton (1971), we know that $\varphi(t, x)$ solves the classic HJB equation

$$
\begin{aligned}
\sup _{(\pi, c) \in \mathbb{R} \times \mathbb{R}_{+}} & \left\{u(t, c)+\varphi_{t}(t, x)-c \varphi_{x}(t, x)+x r^{*} \varphi_{x}(t, x)(1-\pi)\right. \\
& \left.+\varphi_{x}(t, x) x \pi \mu^{*}+\frac{1}{2} x^{2} \varphi_{x x}(t, x) \pi^{2} \bar{\sigma}^{2}\right\}=0 .
\end{aligned}
$$

and the optimal policy is given by $\pi^{*}=\frac{\mu^{*}-r^{*}}{\alpha \bar{\sigma}^{2}}=1$ and $c^{*}=\left[K^{\alpha^{-1}} e^{\beta \alpha^{-1}(T-t)}+\right.$ $\left.\alpha(\beta-\delta)^{-1} e^{-\delta \alpha^{-1} t}\left(e^{(\beta-\delta) \alpha^{-1}(T-t)}-1\right)\right]^{-1} x \exp \left(-\delta \alpha^{-1} t\right)$. Note that $\mu^{*}, \bar{\sigma}$ and $r^{*}$ and $\varphi$ also solve the HJBI equation (3.4).

Let $(\pi, c)$ be an admissible policy and let $X$ be the corresponding wealth process. By Itô's lemma under $P^{\left(\mu^{*}, \bar{\sigma}, r^{*}\right)} \in \mathcal{P}$, we have

$$
\begin{aligned}
d M_{t}^{\pi, c}= & \left(u\left(t, c_{t}\right)-c_{t} \varphi_{x}\left(t, X_{t}^{(\pi, c)}\right)+\varphi_{t}\left(t, X_{t}^{(\pi, c)}\right)+\varphi_{x}\left(t, X_{t}^{(\pi, c)}\right)\left(r^{*} X_{t}^{(\pi, c)}\right.\right. \\
& \left.\left.+\pi_{t} X_{t}^{(\pi, c)}\left(\mu^{*}-r^{*}\right)\right)+\frac{1}{2} \pi_{t}^{2}\left(X_{t}^{(\pi, c)}\right)^{2} \bar{\sigma}^{2} \varphi_{x x}\left(t, X_{t}^{(\pi, c)}\right)\right) d t \\
& +\varphi_{x}\left(t, X_{t}^{(\pi, c)}\right) \pi_{t} \sigma X_{t}^{(\pi, c)} d W_{t}^{\mu^{*}, \bar{\sigma}, r^{*}} \\
\leq & \varphi_{x}\left(t, X_{t}^{(\pi, c)}\right) \pi_{t} \bar{\sigma} X_{t}^{(\pi, c)} d W_{t}^{\mu^{*}, \bar{\sigma}, r^{*}}
\end{aligned}
$$

and we conclude that $M^{\pi, c}$ is a $P^{\left(\mu^{*}, \bar{\sigma}, r^{*}\right)}$-supermartingale.

Let us write $g(t, \mu, \sigma, r)=u\left(t, c_{t}\right)-c_{t} \varphi_{X}\left(t, X_{t}^{(\pi, c)}\right)+\varphi_{t}\left(t, X_{t}^{(\pi, c)}\right)$ $+\varphi_{x}\left(t, X_{t}^{(\pi, c)}\right)\left(r X_{t}^{(\pi, c)}+\pi_{t} X_{t}^{(\pi, c)}(\mu-r)\right)+\frac{1}{2} \pi_{t}^{2}\left(X_{t}^{(\pi, c)}\right)^{2} \sigma^{2} \varphi_{x x}\left(t, X_{t}^{(\pi, c)}\right)$ for the drift term of $M^{\pi, c}$, see above. Then

$$
\begin{aligned}
& \inf _{P \in \mathcal{P}} E_{P}\left[\int_{0}^{T} u\left(s, c_{s}\right) d s+\Phi\left(T, X_{T}^{(\pi, c)}\right)\right] \\
& =\inf _{(\mu, \sigma, r) \in \Theta} E_{P^{(\mu, \sigma, r)}}\left[\int_{0}^{T} u\left(s, c_{s}\right) d s+\Phi\left(T, X_{T}^{(\pi, c)}\right)\right] \\
& =\inf _{(\mu, \sigma, r) \in \Theta} E_{P^{(\mu, \sigma, r)}}\left[M_{T}^{(\pi, c)}\right] \\
& =\inf _{(\mu, \sigma, r) \in \Theta} E_{P^{(\mu, \sigma, r)}}\left[\int_{0}^{T} g\left(t, \mu_{t}, \sigma_{t}, r_{t}\right) d t\right]+\varphi\left(0, x_{0}\right) \\
& \geq \inf _{(\mu, \sigma, r) \in \Theta} E_{P^{(\mu, \sigma, r)}}\left[\int_{0}^{T} \inf _{(\mu, \sigma, r) \in \Theta} g\left(t, \mu_{t}, \sigma_{t}, r_{t}\right) d t\right]+\varphi\left(0, x_{0}\right) .
\end{aligned}
$$

Thanks to the fact that $\mu^{*}, \bar{\sigma}$ and $r^{*}$ and $\varphi$ also solves the HJBI equation (3.4), we have

$$
\inf _{P \in \mathcal{P}} E_{P}\left[\int_{0}^{T} u\left(s, c_{S}\right) d s+\Phi\left(T, X_{T}^{(\pi, c)}\right)\right]
$$




$$
\begin{aligned}
& \geq \inf _{(\mu, \sigma, r) \in \Theta} E_{P^{(\mu, \sigma, r)}}\left[\int_{0}^{T} g\left(t, \mu^{*}, \bar{\sigma}, r^{*}\right) d t\right]+\varphi\left(0, x_{0}\right) \\
& =E_{P^{\left(\mu^{*}, \bar{\sigma}, r^{*}\right)}}\left[\int_{0}^{T} g\left(t, \mu^{*}, \bar{\sigma}, r^{*}\right) d t\right]+\varphi\left(0, x_{0}\right) \\
& =E_{P^{\left(\mu^{*}, \bar{\sigma}, r^{*}\right)}}\left[\int_{0}^{T} u\left(s, c_{S}\right) d s+\Phi\left(T, X_{T}^{(\pi, c)}\right)\right] .
\end{aligned}
$$

On the other hand, we have obviously

$$
\begin{aligned}
& \inf _{P \in \mathcal{P}} E_{P}\left[\int_{0}^{T} u\left(s, c_{S}\right) d s+\Phi\left(T, X_{T}^{(\pi, c)}\right)\right] \\
& \quad \leq E_{P^{\left(\mu^{*}, \bar{\sigma}, r^{*}\right)}}\left[\int_{0}^{T} u\left(s, c_{S}\right) d s+\Phi\left(T, X_{T}^{(\pi, c)}\right)\right] .
\end{aligned}
$$

Therefore,

$$
\begin{aligned}
& \inf _{P \in \mathcal{P}} E_{P}\left[\int_{0}^{T} u\left(s, c_{S}\right) d s+\Phi\left(T, X_{T}^{(\pi, c)}\right)\right] \\
& \quad=E_{P^{\left(\mu^{*}, \bar{\sigma}, r^{*}\right)}}\left[\int_{0}^{T} u\left(s, c_{s}\right) d s+\Phi\left(T, X_{T}^{(\pi, c)}\right)\right] .
\end{aligned}
$$

We now show that $M^{\pi^{*}, c^{*}}$ is a $P^{\left(\mu^{*}, \bar{\sigma}, r^{*}\right)}$-martingale. In fact, for the choice $\pi^{*}$ and $c^{*}$, the drift term of $M^{\pi^{*}, c^{*}}$ vanishes, i.e.

$$
\begin{aligned}
d M_{t}^{\pi^{*}, c^{*}}= & \left(u\left(t, c_{t}^{*}\right)-c_{t}^{*} \varphi_{x}\left(t, X_{t}^{\left(\pi^{*}, c^{*}\right)}\right)+\varphi_{t}\left(t, X_{t}^{\left(\pi^{*}, c^{*}\right)}\right)+\varphi_{x}\left(t, X_{t}^{\left(\pi^{*}, c^{*}\right)}\right)\left(r^{*} X_{t}^{\left(\pi^{*}, c^{*}\right)}\right.\right. \\
& \left.\left.+\pi_{t} X_{t}^{\left(\pi^{*}, c^{*}\right)}\left(\mu^{*}-r^{*}\right)\right)+\frac{1}{2}\left(\pi_{t}^{*}\right)^{2}\left(X_{t}^{\left(\pi^{*}, c^{*}\right)}\right)^{2} \bar{\sigma}^{2} \varphi_{x x}\left(t, X_{t}^{\left(\pi^{*}, c^{*}\right)}\right)\right) d t \\
& +\varphi_{x}\left(t, X_{t}^{\left(\pi^{*}, c^{*}\right)}\right) \pi_{t}^{*} \bar{\sigma} X_{t}^{\left(\pi^{*}, c^{*}\right)} d W_{t}^{\mu^{*}, \bar{\sigma}, r^{*}} \\
= & \varphi_{x}\left(t, X_{t}^{\left(\pi^{*}, c^{*}\right)}\right) \pi_{t}^{*} \bar{\sigma} X_{t}^{\left(\pi^{*}, c^{*}\right)} d W_{t}^{\mu^{*}, \bar{\sigma}, r^{*}} .
\end{aligned}
$$

The conditions of Theorem 1 are thus satisfied and the proof is done.

In the remainder of this section, we provide the optimal portfolio for all parameter cases that we did not study above.

Theorem 3 (i) For $\bar{\mu} \leq \underline{r}$, the optimal portfolio choice is

$$
\pi^{*}=\frac{\bar{\mu}-\underline{r}}{\alpha \bar{\sigma}^{2}}
$$

(ii) For $\underline{\mu}<\underline{r}<\bar{\mu}$, the optimal portfolio choice is $\pi^{*}=0$. 


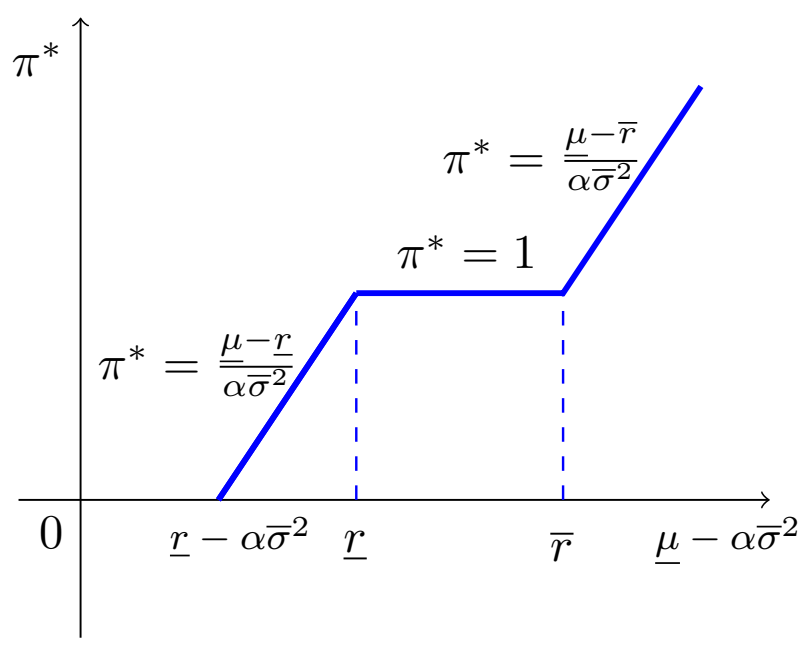

Fig. 1 We illustrate our main finding for the case $\underline{\mu} \geq \underline{r}$. We plot the optimal portfolio as a function of the variable $x=\mu-\alpha \bar{\sigma}^{2}$. As long as $x \in[\underline{r}, \bar{r}]$, the investor puts all the wealth in the risky asset, i.e. $\pi^{*}=1$. If $x>\bar{r}$, the investor uses the familiar Merton portfolio for the worst case parameters $\underline{\mu}, \bar{r}$, and $\bar{\sigma}$

(iii) For $\underline{\mu} \geq \underline{r}$, the optimal portfolio choice is

$$
\pi^{*}=\left\{\begin{array}{l}
\frac{\underline{\mu}-\underline{r}}{\alpha \bar{\sigma}^{2}}, \text { if } \underline{r}-\alpha \bar{\sigma}^{2} \leq \underline{\mu}-\alpha \bar{\sigma}^{2}<\underline{r} ; \\
\frac{\mu-\bar{r}}{\alpha \bar{\sigma}^{2}}, \text { if } \underline{\mu}-\alpha \bar{\sigma}^{2} \geq \bar{r} .
\end{array}\right.
$$

The proof runs along the same lines as the proof of Theorem 2 above; one has to identify the worst case parameters for interest rate, volatility, and drift. The one uses the value function of the original Merton problem for those candidate worst case parameters, and one finally has to verify that the (super)martingale conditions of Theorem 1 hold true.

If the risky asset is known to be dominated by bonds in the sense that the highest expected return $\bar{\mu}$ is below the lowest possible interest rate, the investor short sells the asset and uses the adapted Merton formula for the portfolio with the worst case parameters highest expected return and lowest possible interest rate. We obtain a generalized version of the Dow-Werlang result if Knightian uncertainty about expected returns dominates the Knightian uncertainty about interest rates (case (ii)). Last not least, if the investor knows that the asset's expected return dominate interest rates, he uses again an adapted Merton portfolio, the worst case interest rate being the lowest one if he saves $\left(\pi^{*} \leq 1\right)$, and the highest one, if he borrows money. Figures 1 and 2 illustrate our findings. 


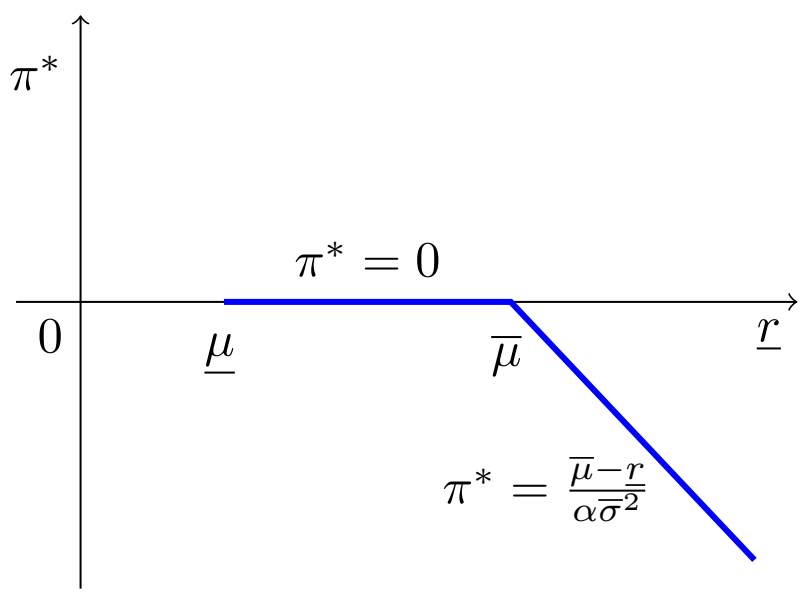

Fig. 2 In this picture, we consider the case $\mu \leq \underline{r}$. We plot the optimal portfolio as a function of $\underline{r}$. As long as $\underline{r} \in[\mu, \bar{\mu}]$, we obtain the known result of Dow-Werlang, i.e. $\pi^{*}=0$. For $\underline{r}<\bar{\mu}$, the investor sells short and uses the familiar Merton portfolio for the worst case parameters $\bar{\mu}, \underline{r}$, and $\bar{\sigma}$

\section{Conclusion}

We study continuous-time consumption and portfolio choice in the presence of Knightian uncertainty about interest rates. For robust parameter sets, the investor puts all his wealth into the asset market when interest rate uncertainty is sufficiently high. Both saving and borrowing are considered to be too uncertain to be worthwhile activities. This insight might have important consequences for policy makers; while central bankers might prefer to remain vague about their future interest rate policies, they should bear in mind that this behavior can have substantial implications for the bond market.

To see that our bounds might be relevant in the real world, take a moderately riskaverse agent with $\alpha=1$. A reasonable upper bound for volatility might be $30 \%$ according to empirical studies (Jiang and Tian 2005), hence $\bar{\sigma}^{2}=0.09$. If the investor assumes a lower bound for returns of $2 \%$, and fears a negative real interest rate (or an inflation) of minus seven percent, he puts all wealth into the stock. With current nominal interest rates being close to zero in developed countries, such a scenario is not too far-fetched for a long term investor as inflation has historically reached levels of $10 \%$ in many countries.

Funding Open Access funding provided by Projekt DEAL.

Open Access This article is licensed under a Creative Commons Attribution 4.0 International License, which permits use, sharing, adaptation, distribution and reproduction in any medium or format, as long as you give appropriate credit to the original author(s) and the source, provide a link to the Creative Commons licence, and indicate if changes were made. The images or other third party material in this article are included in the article's Creative Commons licence, unless indicated otherwise in a credit line to the material. If material is not included in the article's Creative Commons licence and your intended use is not permitted by statutory regulation or exceeds the permitted use, you will need to obtain permission directly from the copyright holder. To view a copy of this licence, visit http://creativecommons.org/licenses/by/4.0/. 


\section{References}

Anderson, E., Hansen, L., Sargent, T.: A quartet of semigroups for model specification, robustness, prices of risk, and model detection. J. Eur. Econ. Assoc. 1(1), 68-123 (2003)

Anscombe, F., Aumann, R.: A definition of subjective probability. Ann. Math. Stat. 34, 199-205 (1963)

Artzner, P., Delbaen, F., Eber, J.-M., Heath, D.: Coherent measures of risk. Math. Finance 9, 203-228 (1999)

Barberis, N.: Investing for the long run when returns are predictable. J. Finance 55, 225-264 (2000)

Biagini, S., Mustafa, P.: The robust Merton problem of an ambiguity averse investor. Math. Financ. Econ. 11, 1-24 (2017)

Chacko, G., Viceira, L.: Dynamic consumption and portfolio choice with stochastic volatility in incomplete markets. Rev. Financ. Stud. 18, 1369-1402 (2005)

Chen, Z., Epstein, L.: Ambiguity, risk and asset returns in continuous time. Econometrica 70, 1403-1443 (2002)

Dow, J., Werlang, S.: Uncertainty aversion, risk aversion, and the optimal choice of portfolio. Econometrica 60(1), 197-204 (1992)

Epstein, L., Ji, S.: Ambiguous volatility and asset pricing in continuous time. Rev. Financ. Stud. 26, 17401786 (2013)

Epstein, L., Schneider, M.: IID: independently and indistinguishably distributed. J. Econ. Theory 113(1), 32-50 (2003a)

Epstein, L., Schneider, M.: Recursive multiple priors. J. Econ. Theory 113, 1-31 (2003b)

Föllmer, H., Penner, I.: Convex risk measures and the dynamics of their penalty functions. Stat. Decis. 24, 61-96 (2006)

Föllmer, H., Schied, A.: Convex measures of risk and trading constraints. Finance Stoch. 6, 429-447 (2002)

Föllmer, H., Schied, A., Weber, S.: Robust preferences and robust portfolio choice. In: Bensoussan, A., Zhang, Q., Ciarlet, P.G. (eds.) Mathematical Modelling and Numerical Methods in Finance, Handbook of Numerical Analysis, vol. 15. Elsevier, Amsterdam (2009)

Gilboa, I., Schmeidler, D.: Maxmin expected utility with non-unique prior. J. Math. Econ. 18, 141-153 (1989)

Hansen, L.P., Sargent, T.J.: Robustness. Princeton University Press, Princeton (2011)

Jiang, G.J., Tian, Y.S.: The model-free implied volatility and its information content. Rev. Financ. Stud. 18, 1305-1342 (2005)

Korn, R., Kraft, H.: A stochastic control approach to portfolio problems with stochastic interest rates. SIAM J. Control Optim. 40, 1250-1269 (2001)

Kraft, H.: Optimal portfolios and Heston's stochastic volatility model. Quant. Finance 5, 303-313 (2005)

Lin, Q., Riedel, F.: Optimal consumption and portfolio choice with ambiguity (2014). arXiv preprint arXiv: 1401.1639

Liu, H.: Robust consumption and portfolio choice for time-varying investment opportunities. Ann. Finance 6, 425-454 (2010)

Liu, H.: Dynamic portfolio choice under ambiguity and regime-switching mean returns. J. Econ. Dyn. Control 35, 623-640 (2011)

Liu, J.: Portfolio selection in stochastic environments. Rev. Financ. Stud. 20, 1-39 (2007)

Luo, Y.: Robustly strategic consumption-portfolio rules with informational frictions. Manag. Sci. 63(12), 4158-4174 (2017)

Luo, Y., Nie, J., Young, E.R.: Ambiguity, low risk-free rates, and consumption inequality. Econ. J. (2020)

Maccheroni, F., Marinacci, M., Rustichini, A.: Ambiguity aversion, robustness, and the variational representation of preferences. Econometrica 74, 1447-1498 (2006a)

Maccheroni, F., Marinacci, M., Rustichini, A.: Dynamic variational preferences. J. Econ. Theory 128, 4-44 (2006b)

Maenhout, P.: Robust portfolio rules and asset pricing. Rev. Financ. Stud. 17, 951-983 (2004)

Merton, R.C.: Lifetime portfolio selection under uncertainty: the continuous-time case. Rev. Econ. Stat. 51, 247-257 (1969)

Merton, R.C.: Optimum consumption and portfolio rules in a continuous-time model. J. Econ. Theory $\mathbf{3}$, 373-413 (1971)

Miao, J.: Ambiguity, risk and portfolio choice under incomplete information. Ann. Econ. Finance 10, 257-279 (2009)

Neufeld, A., Nutz, M.: Robust utility maximization with Lévy processes. Math. Finance 28(1), 82-105 (2018) 
Quenez, M.-C.: Optimal portfolio in a multiple-priors model. In: Seminar on Stochastic Analysis, Random Fields and Applications IV, pp. 291-321. Springer (2004)

Revuz, D., Yor, M.: Continuous Martingales and Brownian Motion. Springer, Berlin (1999)

Riedel, F.: Dynamic coherent risk measures. Stoch. Process. Appl. 112, 185-200 (2004)

Savage, L.: The Foundations of Statistics. Wiley, New York (1954)

Schied, A.: Optimal investments for robust utility functionals in complete market models. Math. Oper. Res. 30, 750-764 (2005)

Schied, A.: Robust optimal control for a consumption-investment problem. Math. Methods Oper. Res. 67, 1-20 (2008)

Trojani, F., Vanini, P.: A note on robustness in Merton's model of intertemporal consumption and portfolio choice. J. Econ. Dyn. Control 26, 423-435 (2002)

Publisher's Note Springer Nature remains neutral with regard to jurisdictional claims in published maps and institutional affiliations. 